\title{
De l'apparition fantomatique à la résurrection glorieuse : les divers visages de la mort dans les Méditations dans un cimetière d'Andreas Gryphius
}

Von der Erscheinung der Gespenster bis zur großartigen Wiederauferstehung: die verschiedenen Aspekte des Todes in Andreas Gryphius' Kirchhofsgedanken From the apparition of ghosts tot he glorious resurrection : the various faces of death in Andreas Gryphius' Meditation in a churchyard

Yves lehl

\section{OpenEdition}

\section{Journals}

Édition électronique

URL : https://journals.openedition.org/ceg/11772

DOI : $10.4000 /$ ceg. 11772

ISSN : 2605-8359

Éditeur

Presses Universitaires de Provence

Édition imprimée

Date de publication : 1 avril 2012

Pagination : 151-163

ISBN : 0751-4239

ISSN : 0751-4239

Référence électronique

Yves lehl, « De l'apparition fantomatique à la résurrection glorieuse : les divers visages de la mort dans les Méditations dans un cimetière d'Andreas Gryphius », Cahiers d'Études Germaniques [En ligne], 62 | 2012, mis en ligne le 12 octobre 2020, consulté le 04 juin 2021. URL : http://journals.openedition.org/ ceg/11772 ; DOI : https://doi.org/10.4000/ceg.11772 


\section{De l'apparition fantomatique à la résurrection glorieuse : les divers visages de la mort dans les Méditations dans un cimetière d'Andreas Gryphius}

Yves IEHL

Université de Toulouse 2 - Le Mirail

L'œuvre littéraire d'Andreas Gryphius, profondément marquée par les convictions religieuses de son auteur, est historiquement antérieure à la lutte menée par les théologiens protestants, au XVIII ${ }^{\mathrm{e}}$ siècle, contre l'attachement dogmatique et populaire aux figures des démons et des spectres, et elle ne marque aucun des signes du mouvement de sécularisation qui a caractérisé le siècle des Lumières. Si elle n'occulte pas la figure du diable ${ }^{1}$, objet de terreur religieuse par excellence, elle ne l'évoque que de façon mesurée, et bien qu'Andreas Gryphius se montre très préoccupé par la pensée de l'au-delà, il se soucie beaucoup plus du devenir de la personne humaine après la mort que des incursions de créatures surnaturelles dans l'univers des vivants. C'est ainsi que les fantômes, que l'on rencontre dans certains de ses drames ${ }^{2}$, sont peu fréquents dans ses poèmes, si ce n'est peut-être sous une forme métaphorique banale. Enfin, malgré la virtuosité rhétorique et stylistique quasi ludique avec laquelle il déploie l'arsenal de ses métaphores et images poétiques ${ }^{3}$, en dépit de l'analogie typiquement baroque et fort suggestive qu'il établit entre l'instabilité du monde terrestre et le jeu d'échecs dans son poème Ebenbild unseres Lebens. Auff das gewöhnliche Königs-Spiel, Andreas

1 Il est présent dans la poésie religieuse notamment, et a inspiré quelques belles expressions poétiques comme "Le prince de la nuit obscure" ("der printz der schwartzen nacht", sonnet des dimanches et jours fériés ${ }^{\circ}$ XXII, Auff den sontag des grossen schlangentreters, oder Oculi, in Andreas GRYPHIUS, Werke in drei Bänden mit Ergänzungsband, Bd. 3, Lyrische Gedichte, Hildesheim, Olms, 1961, p. 37) et les "semailles de Satan" ("Des satans saat", sonnet $\mathrm{n}^{\circ} \mathrm{XV}$, Auff den sontag des langmütigen ackermanns, ibid., p. 32).

2 C'est notamment le cas dans Cardenio et Celinde et Le fantôme amoureux (Verlibtes Gespenste, Gesang-Spil).

3 Volker MEID évoque notamment, parmi les particularités du style lyrique de Gryphius, son talent à nommer les objets en accumulant et diversifiant les métaphores d'une façon répétitive et insistante, in Walter HINDERER, Geschichte der deutschen Lyrik. Vom Mittelalter bis zur Gegenwart, Würzburg, Königshausen und Neumann, 2001, p. 95. 
Gryphius se montre, au regard de tout ce qui concerne le salut, l'au-delà et les fins dernières de notre existence, fort peu enclin au jeu littéraire. Et pourtant un de ses textes les plus singuliers, parfois considéré avec scepticisme par la critique et qui témoigne d'une façon assez inhabituelle de l'importance essentielle que son auteur, qui a écrit de nombreux poèmes et discours funèbres, accordait au thème de la mort, présente à travers la description d'un ballet de défunts surgis de leurs tombes, un tableau fantastique et halluciné de la présence de la mort dans la vie. Les méditations dans un cimetière, publiées en 1657 et dont Andreas Gryphius a puisé l'inspiration dans certaines odes du jésuite Jacob Balde, qu'il a traduites du latin, constituent une meditatio mortis d'un genre un peu particulier. Partageant à la fois le caractère halluciné des visions ${ }^{4}$ de Jacob Balde et leur finalité édifiante, ce long poème de cinquante octains s'inscrit dans la tradition du memento mori ${ }^{5}$, dont se sont abondamment nourris la littérature et l'art baroques.

En même temps, il se distingue nettement des œuvres de la même veine au $\mathrm{XVII}^{\mathrm{e}}$ siècle, qui pourtant ne dédaignait pas le spectacle de la mort, par l'intensité macabre d'une vision qui se plaît à explorer les conséquences physiques de la mort et les formes de la décomposition, avant de se clore sur une scène de Jugement dernier précédée, conformément à la théorie augustinienne, d'une résurrection corporelle intégrale des défunts. Les interprétations de cette œuvre, surtout envisagée dans l'optique de sa vocation édifiante et en relation avec les principaux thèmes de la pensée chrétienne qu'elle véhicule ${ }^{6}$, n'ont pas conclu à une originalité particulière. On peut cependant se demander pourquoi ce poème accorde une telle place au registres du macabre et de l'horrible, et quelle place y revient au fantastique. Nous envisagerons donc la valeur et le rôle que ces éléments y acquièrent, et la façon dont ils s'articulent avec la perspective religieuse de l'œuvre, qui est a priori éloignée de toute sécularisation.

Les commentateurs ont été le plus souvent heurtés par la brutalité de l'évocation de la mort. Dans sa préface aux Méditations de l'édition complète de 1884 rééditée en 1961, Hermann Palm s'en émeut visiblement :

On ne peut nier que Czepko a traité le même thème avec plus de goût que Gryphius qui, dans ses images hideuses et horribles, va plus loin que dans aucune de ses autres œuvres lyriques. L'époque terrible qui a inspiré ces

4 Ferdinand VAN INGEN attribue à la "vision" la valeur sinon d'un genre, du moins d'une catégorie à part entière de la littérature baroque. In Vanitas und Memento Mori in der deutschen Barocklyrik, Groningen, Wolters, 1966, p. 266-275.

5 Johann Anselm STEIGER aperçoit dans ce texte "une adaptation poétique de grande ampleur du memento mori", in Schule des Sterbens. Die Kirchhofsgedanken des Andreas Gryphius als poetologische Theologie im Vollzug, Heidelberg, Palatina, 2000, p. 31.

6 Cf. notamment Günter OTT, Die vier letzten Dinge in der Lyrik des Andreas Gryphius. Untersuchungen zur Todesauffassung des Dichters und zur Tradition des eschatologischen Zyklus, F. a M., 1985, p. 284. Cet auteur étudie le thème des fins dernières de l'existence dans l'œuvre lyrique de Gryphius avec un zèle théologique admirable et souligne avec raison la visée édifiante des Méditations mais se pose moins la question de la spécificité et de l'originalité de sa création dans une optique proprement littéraire. 
Méditations dans un cimetière explique partiellement l'horrible succession de tableaux (strophes 27 à 31 ) dont l'imagination du poète se délecte littéralement $[\ldots]$. $^{7}$

Plus sobrement, Günter Ott souligne - sans se poser la question des motivations de l'auteur - le "mauvais goût [...] d'un point de vue artistique" 8 d'une partie du poème tout en se demandant si, à travers cette œuvre, Andreas Gryphius ne déroge pas aux exigences de l'esthétique baroque en régressant vers une rudesse d'un autre âge et en cédant à "une joie médiévale à fouiller la pourriture" $"$. Dans une optique très différente, le théologien protestant Johann Anselm Steiger, auteur d'un ouvrage assez récent sur les Méditations dans un cimetière, situe celles-ci dans le sillage immédiat de la pensée de Luther, qui défend, dans certains de ses prêches, l'idée que la pensée de la mort et de la décomposition, dans la vie, est essentielle pour renforcer, à l'approche de la mort, la foi dans la vie éternelle. Il s'efforce ainsi de justifier le parti pris esthétique adopté par Andreas Gryphius en suggérant qu'il est dû à son adhésion à la vision du réformateur de Wittenberg, avançant du reste que celui-ci a fondé dans certains de ses prêches une "esthétique de la répulsion" $"$. Cependant la perspective théologique exclut le plus souvent une prise en compte véritable de la dimension proprement littéraire de l'œuvre.

La diversité et la pertinence relative de ces arguments et perspectives ne masque pas un certain désarroi devant la singularité d'un texte inhabituel et dérangeant. Les Méditations se distinguent tout d'abord par le site original dans lequel elles se déroulent et qui les inspire, un locus de morte ${ }^{11}$ par excellence, lieu transitionnel pour les défunts dans l'attente du Jugement dernier, et de préparation à la mort pour les vivants. Vivement apprécié et recommandé comme endroit de recueillement et de prière aussi bien par Luther $^{12}$ que par Aegidius Albertinus ${ }^{13}$, figure célèbre de la Contre-Réforme, le cimetière, un des topos classiques de la meditatio mortis ${ }^{14}$, est recherché par le moi lyrique, ici très proche de l'auteur, pour le "calme" et le

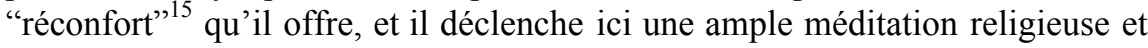

7 Herrmann PALm (Hg.), Kirchhofs-Gedanken, Vorwort des Herausgebers, in A. GRYPHIUS, Lyrische Gedichte, p. 336.

8 Günter OTT, Die vier letzten Dinge, p. 283.

9 Ibidem, p. 137.

10 J. A. STEIGER, Schule des Sterbens, p. 50 ss.

11 Ibid., p. 40.

12 J. A. STEIGER, ibid.., p. 37, cite Luther (WA 23, 375, 28-34, "Ob man vor dem Sterben fliehen möge" 1527) et aperçoit dans les Méditations dans un cimetière une application poétique des recommandations du réformateur.

13 Cf. G. OTT, Die vier letzten Dinge, p. 280, qui cite sans doute un extrait assez long de Der Seelen Compaß, München, Nicolaus Henricus, MDCXVII.

14 On songe bien sûr aux odes de Jacob Balde, mais G. OTT, ibid., p. 280, cite également des œuvres plus anciennes de Engelberger et Hugo von Monfort.

15 Andreas GRYPHIUS, Kirchhofs-Gedanken, in Lyrische Werke, p. 340 : "Hat ruh' erquickung hier bestellt / Dem, der sich für und für bemühet, (...)”. Ce texte sera désormais cité dans le corps du texte d'après cette édition, les citations étant suivies de l'indication, entre parenthèses, des numéros de strophe, de vers et de page. 
poétique. Par son austère gravité, ce lieu de mort apparaît comme l'antithèse tout à la fois de l'agitation humaine et sociale, et du règne végétal. Avec un très vif sens de l'antithèse, Gryphius vante son âpre beauté tout en revendiquant avec véhémence son aversion pour l'art des jardins ("Mir graut vor aller gärte zier !", 2, 13,340), confirmant ainsi également le peu de goût qu'il éprouve habituellement pour la nature ${ }^{16}$. Il exprime en outre son dégoût de ces symboles de la beauté végétale exotique que sont la rose, le jasmin, la tulipe, la fleur de la passion $(3,18-22,340)$, fleurs élégantes et odorantes qu'il associe au mirage de la beauté terrestre fugace qui détourne l'homme de la pensée de la mort. Par sa sobriété, l'évocation de ce cimetière, dont la description est fort discrète, fait songer à la solitude désolée du sonnet Solitude ${ }^{17}$.

La finalité de cette méditation est bien sûr édifiante, comme l'exprime explicitement la répétition anaphorique de l'exclamation solennelle en forme d'invocation ("O Schul!"), par laquelle débutent les strophes 4 à 7 , car elle vise à inciter le lecteur au retour à une foi authentique et à la renonciation aux biens et réalités terrestres (S 5) qui s'interposent entre lui et le salut. Assez typiquement baroque, une telle intention est néanmoins formulée avec une intensité et une insistance sensibles, l'attente et la quête pathétique d'un enseignement moral s'associant au désir tout aussi poignant de le transmettre au lecteur. Gryphius se pose en quelque sorte en exemple : par la voix du protagoniste, le poeta laureatus prend ses distances avec la littérature et la philosophie (S 4 et 7) dont il affirme percer à jour la vanité, puis il s'attache ensuite à faire ressortir, à l'attention des pécheurs endurcis, la menace de l'enfer (S 6), ici fortement dramatisée par l'expérience du cimetière.

Mais cette partie du poème a aussi et surtout pour fonction de préparer l'irruption de la vision macabre, d'autant plus impressionnante et spectaculaire qu'elle n'est aucunement annoncée, bien que la strophe 8 puisse en un sens faire figure de transition masquée, et l'effet de surprise est total.

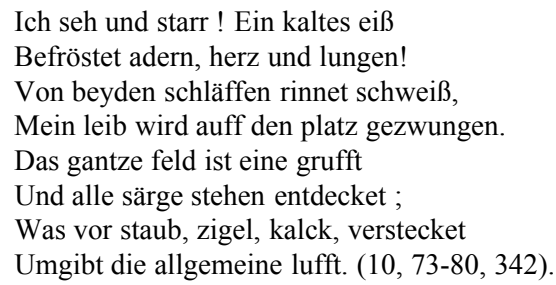

16 Adolf STRUTZ note que l'on ne rencontre, dans les poèmes de Gryphius, pas de véritable enthousiasme envers les formes réelles, végétales, animales ou paysagères de la nature, in Andreas Gryphius, Die Weltanschauung eines deutschen Barockdichters, Leipzig, 1931, p. 58 ss.

17 C'est ce que suggère le premier quatrain : "In dieser einsamkeit der mehr denn öden wüsten / Gestreckt auf wildes kraut, an die bemooste see, / Beschau ich jenes thal und dieser felsen höh' / Auf welchen eulen nur und stille vögel nisten", in A. GRYPHIUS, Lyrische Werke, p. 131. 
Le texte acquiert alors une dimension fantastique, suggérée à travers l'effroi palpable du protagoniste de ce récit en vers, et le fait que l'événement se déroule en deux étapes révèle une volonté délibérée de mise en scène et de dramatisation croissante. Nous assistons tout d'abord à un véritable tremblement de terre, bouleversement tellurique inexplicable et inexpliqué, qui exhume les cercueils (S 9 à 14). La vue de ceux-ci suscite une série de développements plus réflexifs sur la naïveté des hommes, qui s'entêtent à les considérer comme des refuges protecteurs ${ }^{18}$ (S 11), puis sur leur vanité, sensible jusque dans la mort (S 12). Ce passage est en fait destiné à ménager un faux répit avant une seconde vision d'horreur, plus éprouvante encore, lorsque les défunts, libérés par l'effritement et la décomposition du bois, quittent leurs tombes et entourent le protagoniste.

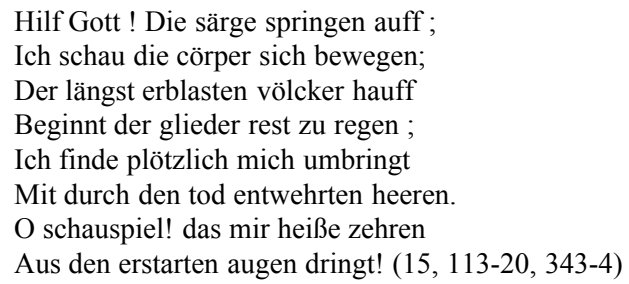

L'atmosphère créée par cette mise en scène est alors incontestablement de l'ordre de l'épouvante et du fantastique, mais ce fantastique ne se développe pas sous la forme à laquelle on serait en droit de s'attendre car la vision ne débouche pas sur une scène de confrontation des morts et des vivants mais reste bien une vision, un spectacle de mort. Par ailleurs la dimension du diabolique et du démoniaque, que l'on aurait pu rencontrer ici et que l'on associe traditionnellement avec la lutte entre les forces du bien et du mal, est totalement absente du poème. Visiblement, ce n'est pas véritablement à une histoire de revenants que l'on a affaire, un genre dont les premières formes dans les pays germaniques émergent à peine au début de l'époque baroque. En revanche, les phénomènes que nous avons évoqués et les visions ultérieures auxquelles ils donnent lieu rappellent la tradition de la danse macabre ${ }^{19}$ qui a, particulièrement en Allemagne, été très féconde. On sait que ce genre pictural et littéraire ${ }^{20}$ de la fin du Moyen Age s'est diffusé dans toute l'Europe, s'est inspiré et nourri du genre du Vado mori, des Dits des trois

18 C'est ce que suggèrent précisément les métaphores, comme toujours très riches de connotations chez Gryphius, de la "maison", du "château", du "palais", à travers lesquelles se cristallisent les espoirs illusoires des vivants de voir leur dépouille protégée des atteintes de toutes sortes $(11,1-4,342-43)$.

19 La façon dont F. v. INGEN évoque la danse macabre invite en effet à un tel rapprochement: "Die Totentanzliteratur strotz förmlich von Verwesungsmotiven", Vanitas, p. 297. Ce rapprochement a du reste déjà été fait par certains commentateurs, mais il exige d'être précisé. Cf. G. OTT, Die vier letzten Dinge, p. 282, et F.-W. WENTZLAFF-EGGEBERT, Der Triumphierende und der besiegte Tod in der Wort- und Bildkunst des Barock. Berlin, de Gruyter, 1975, p. 91.

20 Cf. André CORVISIER, Les danses macabres, Paris, PUF, 1988. 
morts et des trois vifs, des Artes moriendi depuis le début du $\mathrm{XV}^{\mathrm{e}}$ siècle jusqu'au XVII ${ }^{\mathrm{e}}$ siècle où il a commencé à décliner. Il a en particulier été popularisé par les fresques des cimetières ${ }^{21}$ et a donné lieu à travers son succès à un véritable engouement pour la mort, une "banalisation du macabre" ${ }^{22}$, dont l'art baroque porte la marque.

Une telle banalisation est sans doute sensible dans les Méditations, mais à la différence des danses macabres, elles n'illustrent pas cette confrontation des morts et des vivants, par l'échange et le dialogue, qui est nécessaire à la réflexion sur la mort et la vanité des biens terrestres. De même, l'élément également indissociable de la danse macabre qu'est l'évocation successive de l'ensemble des conditions et situations sociales, envisagées dans une perspective critique et satirique ${ }^{23}$, n'y joue qu'un rôle secondaire. Ce ne sont pas ici les défunts qui viennent s'adresser aux vivants, tels des porte-parole de la mort, pour les solliciter, c'est au contraire le protagoniste qui, en proie à une vision hallucinée des morts revenus à la vie, s'efforce de retrouver en eux les traces de leur passé, de leurs identités sociales. Sa tâche est cependant des plus délicates. Dans les strophes 16 et 20, il parvient tout au plus à identifier d'anciens hommes de pouvoir et gens de guerre, puis à départager les plus fortunés des miséreux. Dans les strophes ultérieures, l'appartenance sociale des défunts ne semble plus décelable mais la qualité de leur piété et le degré de leur culpabilité morale permettent au protagoniste de distinguer plusieurs groupes, celui des pécheurs repentants mais lourdement coupables tout d'abord (S 17), ensuite celui des criminels et réprouvés ayant déclenché la colère divine (S 18), enfin celui des êtres purs et justes (S 19). La dimension eschatologique, plus précisément la réflexion sur le salut de l'âme semble ainsi déjà s'esquisser, mais la vision s'oriente bientôt, à partir de la strophe 21 , vers une observation à la fois aiguë et méditative des conséquences physiques de la mort sur le corps. Cette évolution du poème peut paraître surprenante. On peut être tenté de l'expliquer par le fait que l'admiration de Gryphius pour Jacob Balde a pu le conduire à associer d'une façon un peu abrupte les motifs principaux des deux odes qu'il a traduites et qui ont inspiré les Méditations, celui du réveil prématuré des morts avant le Jugement dernier $^{24}$ et celui des formes de la décomposition ${ }^{25}$. Mais une telle évolution reflète aussi l'esthétique particulière du genre de la vision baroque, contemplative et spirituelle, mais aussi fort imaginative, voire hallucinée.

Cette dimension visionnaire se confirme à travers la singularité et l'intensité remarquables que ce poème parvient à conférer à la représentation

21 Les cimetières les plus connus et ornés de ce genre de fresques sont celui, aujourd'hui détruit, des Saints-Innocents à Paris, celui de l'abbaye de La Chaise-Dieu en Auvergne, celui de la Marienkirche de Lübeck, celui de l'église des Prêcheurs de Bâle, celui de la Dreikönigskirche à Dresde.

22 A. CORVISIER, p. 48

23 Ibid., p. 23.

24 Cf. Jacob Balden s. J. verzückung auf dem kirchhof. An dem gedächtnis-tage der selig verstorbenen, in A. GRYPHIUS, Lyrische Werke, p. 355, vers 85 ss.

25 Ibid., p. 358, vers 60-84. 
de la mort, alors même que celle-ci est pourtant un des lieux communs les plus courants de l'art et la littérature baroques et que, en dépit de l'évolution du goût artistique dans le sens d'un plus grand raffinement, la peinture des formes de la corruption physique reste encore fréquente à cette époque. Traditionnellement, à cette époque notamment, la mort est très souvent envisagée sous l'angle de la soudaineté comme une interruption brutale, définitive et irrémédiable du cours de la vie, inscrite, quant à elle, dans la durée. Cette dimension événementielle associée à l'idée de coupure et de rupture, mais aussi de seuil et de passage, lui confère communément les attributs de la fixité et de l'inertie. Elle est perçue comme un état stable et comme le contrepoint antithétique de l'ambivalence de la vie, processus complexe et mouvant qui associe d'une façon indistincte croissance et dégénérescence, vie et mort, bien et mal. Comme le souligne F.-W. Wentzlaff-Eggebert, "seule la mort est une certitude éternelle dans l'incertitude de la vie" "26. Gryphius insiste quant à lui sur un tout autre aspect qu'il met en relief en détournant le potentiel fantastique que présentait l'apparition des défunts sortis de leurs tombes, pour le transférer sur l'image de la mort elle-même. Il insiste non pas sur l'inéluctabilité mais sur la durée et la progressivité du phénomène de désagrégation du corps et de la chair, et observe le déroulement de ce processus, étape par étape, avec une impitoyable rigueur. La puissance suggestive du tableau de délabrement et de décomposition physiques qu'il dépeint, sous son aspect évolutif ou plutôt involutif, réfute l'image d'une mort purement statique et réduite à l'inertie, et montre qu'elle n'est certes rien d'autre que le pouvoir d'autodestruction, inscrit au cœur de l'existence, que l'époque baroque ne cesse de dénoncer, mais sous une forme superlative et paroxystique qui en amplifie considérablement l'image.

Avec une méticulosité quasi scientifique qui rappelle l'intérêt très vif manifesté par l'auteur, au cours de ses années d'étude à l'université de Leyde, pour l'anatomie et la dissection ${ }^{27}$, le protagoniste évoque, dans les strophes 22 à 26, le déroulement inexorable d'un processus de dissociation, de dislocation, de désorganisation de l'image du corps réduit à l'état de squelette décharné, ce même corps dont les artistes, depuis la Renaissance, s'étaient efforcés de redéfinir les proportions et la silhouette idéales. L'observation de ses diverses parties du haut vers le bas (tête, puis torse, membres supérieurs, bassin et jambes) présente, dans sa successivité, une dimension systématique

26 F.-W. WENTZLAFF-EGGEBERT, p. 75.

27 Gryphius avait organisé à Leyde un "collège d'anatomie pratique" et il participa le 16 décembre 1658 à Breslau, un an après la publication des Méditations, à la dissection d'une momie égyptienne, dont il fit le compte-rendu dans Mumiae Wratislavienses, qui fut publié en 1662. Cf. Eberhard MANNACK, Andreas Gryphius, Stuttgart, Metzler, 1986, p. 10 ss. et 104 ss.. $\mathrm{Du}$ reste les allusions précises, dans la strophe 32, à des substances utilisées pour l'embaumement (Aloe socotrina et myrrhe) confirment l'existence chez lui d'un intérêt très vif pour les techniques de momification, dont témoignent aussi ses lectures des textes de l'antiquité consacrées à ce sujet et de Oedipus Aegyptiacus, l'ouvrage d'égyptologie d'Athanase Kircher. 
et une rigueur ${ }^{28}$ qui, alliées au caractère impersonnel et presque clinique du propos, soulignent la régularité et la puissance du phénomène de destruction physique.

Ce tableau est complété par les connotations que chaque strophe ajoute comme pour souligner un aspect particulier du processus général. A l'aspect de la privation, de la déperdition, de la destruction, suggéré par l'utilisation répétée du terme sonder dans la strophe 22, et qui fait songer à une mutilation systématique et délibérée,

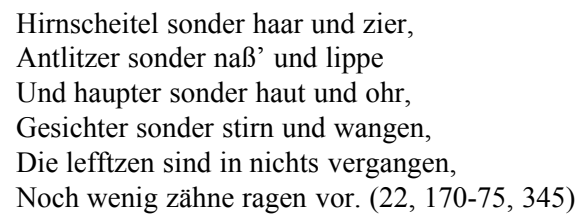

s'associent celles de la déstructuration et de la perte de cohésion, ainsi que de l'anéantissement de la substance organique ("Die eingeweide sind verzehret, / Verzehret des busens doppel-lust" (23, 182-84, 346). La dimension grotesque s'affirme ensuite, tout d'abord à travers la déchéance de la main, emblème par excellence de l'activité et du génie humains, qui apparaît ici disjointe et disloquée,

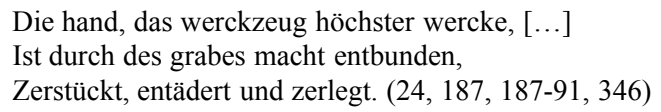

et surtout à travers l'aspect de la difformité, voire de la disparition de toute forme humaine, qui, poussant la désindividualisation jusqu'à l'indétermination, parachève le nivellement produit par la mort.

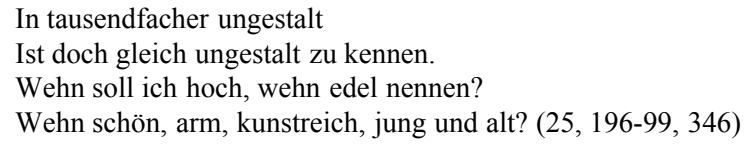

L'art de Gryphius est ici de fournir une illustration magistrale du motif traditionnel de l'égalisation que produit la mort - omnia mors aequat ${ }^{29}$-, qu'il cite du reste expressément dans les Méditations ("Sie zeigt dir [...] / Dass, ob wir hier nicht gleiche sind, / Der tod doch alle gleiche mache", 48, 367-381, 352), tout en parvenant à offrir une image multiple, diverse et nuancée de celle-ci. La dimension du fantastique est ici étroitement corrélée à

28 G. OTT, p. 277-278, évoque à juste titre que ce mode de description est inspiré sans doute par la persistance d'une mode de pensée scolastique et fait songer à une démarche d'énumération encyclopédique mais sur le mode d'un blason inversé qui égrènerait la somme des laideurs physiques du corps. II.

29 Lieu commun du monde antique formulé par Claudien dans L'enlèvement de Proserpine 
celle du grotesque, un grotesque de la désorganisation et du délabrement de la figure humaine, mais qui ne présente pas cette association d'horreur et de naïveté ludique que l'on pouvait rencontrer dans les formes les plus anciennes des genres macabres, un grotesque plus systématique, presque pédant dans son illustration fidèle des effets dévastateurs du péché originel. Mais ce qui est plus spécifiquement fantastique, c'est ici la qualité d'une vision irréelle, intense et hallucinée, certes mise au service d'une intention édifiante mais qui semble, par sa vigueur, dépasser le cadre de celle-ci.

Les strophes suivantes évoquent non plus la désorganisation du squelette, mais la décomposition du corps, et c'est sans doute cette partie de l'œuvre que l'on peut associer le plus directement à la rudesse médiévale de la danse macabre $^{30}$. Il n'est également pas inutile de rappeler que les circonstances qui ont entouré la rédaction des Méditations, et notamment l'épidémie de peste qui sévissait alors en Silésie ${ }^{31}$, ont pu exercer une influence sur cette évocation. Bien que l'on ait pu suspecter l'auteur de vouloir malmener la sensibilité du lecteur, on ne peut manquer de remarquer l'habileté avec laquelle, après avoir illustré dans les strophes 22 à 26 un phénomène de dévitalisation suggéré par la sécheresse des cadavres squelettiques, il réintroduit ensuite au contraire dans l'image de la mort un semblant de vie aussi ambivalent qu'inquiétant. Il y parvient en suggérant que les effets de la décomposition sur une chair dotée encore de l'apparence du vivant (chute des cheveux, mouvements étranges des pupilles, décoloration de la peau, distorsion des chairs rendues grimaçantes, déplacements et glissements, impression de débâcle) s'apparentent à une forme d'animation fantastique qui confère à la vision toute sa vigueur.

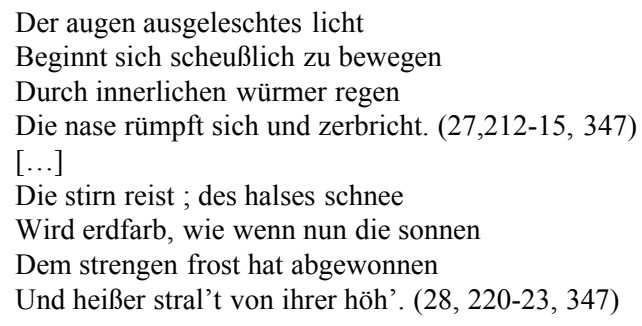

Les bruissements suspects et odeurs fétides qui émanent des corps semblent également doter ceux-ci d'une vie autonome, et le paroxysme est atteint dans la strophe 31 où les ventres éclatent sous l'effet du "grouillement insatiable" $(31,246,348)$ de la vermine. La tonalité dominante est ici celle d'un grotesque intentionnellement repoussant, un grotesque de la putréfaction et de la purulence, qui permet de situer précisément à quel niveau se situe le

30 Cf. note 17.

31 Lorsqu'il écrivit ce texte en 1656, Gryphius s'était retiré avec sa famille dans la propriété de son ami et ancien élève Johann Christoph Schönborn pour fuir la peste qui faisait rage dans toute la région. 
fantastique. Il n'est plus suscité par l'apparition surnaturelle des cadavres, motif rapidement abandonné, mais est lié à une forme interne d'animation et d'autonomisation de la mort, qui résulte de l'intensité et de la radicalité de son pouvoir de transformation et de destruction de la chair. Dans ces dernières strophes, la mort s'apparente à un vertige, un tourbillon, dont on peut se demander si elle ne suscite pas chez l'auteur une fascination muette. C'est ce que suggère par exemple le soin qu'il apporte à amplifier et à intensifier son propos par la référence à des lieux, géographiques ou mythiques, comme le lac Averne, près de Naples, le marécage de Camarina en Sicile et, plus platement, les "grottes des dragons jaunes" (30, 233, 247), motifs auxquels l'imaginaire du XVII ${ }^{\mathrm{e}}$ siècle prêtait visiblement une connotation infernale de fermentation et de pestilence ${ }^{32}$.

Après l'intensité de ces derniers tableaux, le poème quitte provisoirement le terrain de la vision pour adopter un tour plus réflexif et introduire la perspective véritablement eschatologique du texte, c'est-à-dire l'évocation, au-delà de la mort proprement dite, de la parousie, de la résurrection des morts et du Jugement dernier. Cette perspective nouvelle n'annule pas mais prolonge et enrichit la dimension visionnaire des Méditations. Car l'apparition initiale, liée à un phénomène de résurrection anarchique qui n'illustre que les conséquences dévastatrices de la mort, n'était pas tant la préfiguration que le contrepoint grotesque et caricatural du tableau qu'elle annonce et dont elle fait ressortir a contrario la dimension grandiose et cosmique, celui de la résurrection authentique et universelle des défunts, œuvre divine qui annule de façon solennelle et monumentale l'œuvre de la mort. Visiblement, Gryphius a eu à cœur, par cet effet de dédoublement et de symétrie, de doter d'une dimension annonciatrice et eschatologique le motif de la résurrection prématurée qui, dans la première ode de Jacob Balde que nous avons citée, pouvait faire figure d'anecdote plaisante et facétieuse, l'auteur invitant les morts indociles à retourner dans leurs tombes ${ }^{33}$. Ce qui est ici tout à fait remarquable n'est pas seulement la stricte conformité du texte de Gryphius à la conception augustinienne de la résurrection des morts, selon laquelle le "corps ressuscité, bien que spiritualisé et immortalisé [est]

32 On trouve en particulier cette évocation suggestive du lac Averne dans le Dictionnaire de Trévoux édité au début du XVIII ${ }^{\mathrm{e}}$ siècle : "AORNE. Aornus. Lac d'Italie entre Pouzzol, \&

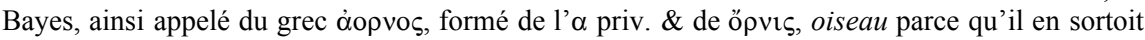
des vapeurs malignes, qui en éloignoient tous les oiseaux, $\&$ faisoient mourir ceux qui passoient par-dessus. Les Poêtes feignoient que c'étoit un lac des enfers. Virgile, Lucrèce \& Claudion l'appellent Averne [...]". In Dictionnaire universel françois et latin, dit de Trévoux, Tome 1, Trévoux, F. Delaulne, 1721, p. 457. Cité d'après http://gallica.bnf.fr/ark:/12148/bpt6k509621/ f256.image

33 Schlafft geister ! haltet inn ! schlafft todten ! haltet innen!

Ich schenck' euch meinen wunsch und dieses thränen-rinnen,

Ich ruff : o ruht, die ihr, was sterblich, habt vollbracht,

Wir sind noch mit im Werck! nun, todten, gute nacht!

Jacob Balden s. J. verzückung auf dem kirchhof, in A. GRYPHIUS, Lyrische Werke, p. 355, vers 93-96. 
absolument identique au corps terrestre ${ }^{34}$, encore que l'auteur insiste tout spécialement sur le souci méticuleux de restauration intégrale de l'intégrité physique des corps humains que manifeste Jésus :

\author{
Er sorgt, dass nicht der meinste staub \\ Von einem cörper ihm verschwinde. \\ Ihm hüten wasser, lufft und winde, \\ Ihm raubt gar nichts der zeiten raub. (49, 388-91, 352)
}

C'est aussi le tableau grandiose de la confrontation du créateur - en la personne de son fils - avec sa propre Création, et l'évocation de la restitution prodigieuse, par celle-ci et par les puissances élémentaires, des corps des défunts résorbés en elles. Ce processus, qui s'apparente à une véritable libération $(41,322-23,350)$, s'opère tout d'abord sur le mode classique de l'ouverture des tombes (S 38) mais fait aussi appel, pour les défunts de plus longue date, à l'élément marin, aux vents (le vent du nord), aux fleuves et contrées exotiques (le Tigre et le Maroc), aux zones climatiques tropicales (les déserts de la Perse), somme toute aux quatre éléments qui apparaissent également impliqués dans ce prolongement logique de la mort qu'est la résorption de la matérialité de la dépouille humaine dans le monde physique. Ce phénomène est du reste mis en relief par son association avec certaines formes de mort violente qui s'apparentent peu ou prou à un processus d'absorption brutale par la matière. C'est notamment perceptible à travers les motifs de l'ingestion cannibalesque des défunts par les peuples anthropophages des Caraïbes et du Brésil, de leur disparition au fond de puits ou de leur ensevelissement sous des cendres ou laves volcaniques, la personnification du Vésuve, de l'Etna, de l'Hekla, ici nommément cités, faisant de ces volcans des entités mythiques dévorantes $(40,312-19,350)$. C'est sans doute le motif de la dispersion dans l'atmosphère $(14,320,350)$ qui offre l'image la plus aboutie de la dématérialisation et de la volatilisation de la chair. Cette dimension mythique, au sens antique et préchrétien du terme, est particulièrement sensible car certains termes métaphoriques (gebären, 39, 305, 350, entbinden, 41, 323, 350, l'allusion, également, au sein d'Amphitrite, 39, 304, 350, qui fait écho au "sein fécond" du cimetière évoqué auparavant, $2,2,340$ ) suggèrent que cette restitution des corps s'apparente à une "naissance", ce qui n'est pas sans rappeler la relation filiale que les hommes de l'Antiquité entretenaient à l'égard de la Terre-Mère. Cependant, ces éléments et connotations qui renvoient au mythe antique sont solennellement et symboliquement surmontés et conjurés, dans ce poème, par le thème chrétien de la résurrection, qui triomphe de la puissance arbitraire des éléments en même temps que de la mort.

C'est donc dans ce double tableau grandiose de l'illustration de la puissance de la mort et de son dépassement inouï par le miracle de la résurrection, que réside l'originalité profonde de ce poème. Nous avons là

34 Jean-Yves Lacoste, Dictionnaire critique de théologie, Paris, PUF, 1988, p. 1207. 
diverses nuances d'un fantastique parfaitement compatible avec la vision chrétienne de l'homme et du monde, et qui exalte celle-ci, le fantastique hallucinant et vertigineux de la décomposition étant contrebalancé par le fantastique cosmique et prodigieux de la restauration des corps, l'homme ressuscité faisant figure d'antithèse glorieuse et triomphale du fantôme. Ce tableau traduit ainsi une préoccupation aiguë du corps, envisagé dans sa réalité physique - on sait à quel point l'expérience personnelle de la maladie, dont les sonnets Thränen in schwerer Kranckheit et An sich selbst sont le reflet, a affecté profondément Gryphius toute sa vie -, et une volonté très remarquable, soutenue par une curiosité quasi scientifique, d'explorer le phénomène et les conséquences de la mort et d'en renouveler la représentation.

Au regard de la puissance de cette évocation, la suite des Méditations, qui s'orientent vers une réflexion sur la destinée de l'âme au terme du Jugement dernier et relèguent la préoccupation de la mort au second plan, paraît au lecteur moderne, en dépit de la cohérence et de l'orthodoxie du propos, présenter moins d'intérêt. En dépit de la tension, voire de la rupture qui s'instaure ici entre la grandiose résurrection des défunts et la terrible incertitude suscitée par l'imprévisibilité du verdict divin, l'évocation des fins dernières de l'humanité est ici conforme à la tradition. Le triomphe du Christ sur la mort n'annule pas le péché et ne préjuge en rien de la destinée des âmes, et nous avons en définitive, magistralement orchestrée à travers une radicalisation impressionnante de l'opposition entre le paradis et l'enfer et dramatisée en outre par le rejet luthérien de l'option du purgatoire, une scène classique de Jugement dernier.

On est tout de même étonné de constater que, bien que l'angoisse fondamentale de la damnation ait toujours tourmenté Gryphius, comme en témoigne son œuvre, le protagoniste ne semble pas, dans les dernières strophes, la partager. La mise en garde solennelle qu'il formule à l'attention de son public est une exhortation dont il semble s'exclure, comme le suggère la position qu'il adopte alors ("Ich werd euch sehn", 45 et 46,352 et 360 , 351 ), celle d'un témoin ou d'un spectateur non concerné par les événements qu'il annonce et commente. Une telle prise de distance, beaucoup plus sensible que dans l'évocation antérieure de la mort, peut être interprétée comme une tentative en vue de conjurer l'angoisse de la damnation par une postulation audacieuse et unilatérale du salut personnel. Plus vraisemblablement cette attitude reflète sans doute la conviction luthérienne que la certitude intérieure de la foi a valeur de justification et sauve par avance le croyant, qui se trouve ainsi exempté de l'épreuve du jugement divin $^{35}$. Il est enfin possible de se demander, compte tenu de la place qu'occupent dans ce poème l'évocation de la mort et surtout celle de son dépassement, dépeint avec une emphase baroque tout à fait remarquable, si

35 C'est dans cette perspective théologique précise que J. A. STEIGER, Schule des Sterbens, p. 60, croit pouvoir interpréter l'attitude du protagoniste dans ce passage. 
dans cette attitude ne transparaît pas la nostalgie secrète d'une apocatastase, d'un salut universel qui abolirait toute angoisse et donnerait alors tout son sens à l'évocation glorieuse de la résurrection des morts. 